\title{
Research on the Functional Foreign Linguistics Based on Complex Adaptive System
}

\author{
Cai Jing, Hong Hong \\ Nanchang Institute of Science \&Technology, Nanchang 330108, China
}

Keywords: Adaptive system, Foreign language, Stimulation channel, Learning memory, Audio-visual sense.

\begin{abstract}
In order to study the functional system of foreign language linguistics, this paper proposes a new method based on adaptive system from the theory perspective of complex adaptive system, and using different stimulation channels can be stimulate the learning of foreign language. The foreign language learning is stimulated by using different stimulation channels, and through the comparison of the auditory, visual and audio-visual, we can find from its memory preservation effect that the audio-visual perception can obtain the amount of language information, to get the best information acquisition mode. Based on complex adaptive system, foreign language can use as a new scientific concept and methodology, which provides a new research model for the study of foreign language linguistics, making linguistics produce complexity science turn, to be more profound understanding the theoretical objectives of foreign language studies.
\end{abstract}

\section{Introduction}

The complex adaptive systems are applied the study of foreign language linguistics, it has brought more inspiration and enlightenment for the researchers, which makes people have a new breakthrough in the evolution of language and complex adaptive system, and provides a new perspective for the field of linguistics [1-3]. Halliday thinks that language is a self-organizing, dynamic and open ecosystem, which follows the law of self-organized criticality. Therefore, only the language is explained as a system, in order to make language and mind, language and design closely linked [4,5]. At the same time, the language is placed in a larger social environment to be able to explain the language phenomenon more systematically, which provides a more extensive research space for the study of language.

\section{The Embodiment of the Complex Adaptive System Theory in the Functional Foreign Linguistics}

The complex adaptive system and functional foreign language are concerned with the dynamic and the behavior of the system. The foreign language learning can be viewed as the interaction between the main body and the elements, but also is a kind of language environment adaptive behavior [6-8]. On the one hand, the functional foreign language system is viewed as the object, it is the system of the system, it can be realized by hierarchical relations, and this hierarchical relationship is expressed as a grade and precision, which forms a complicated dynamic foreign language network system; on the other hand, this theory can be regarded as a kind of mechanism and system, which embodies the foreign language's social meaning and function [9-11]. The adaptive process of foreign language linguistics is shown in Figure 1. 


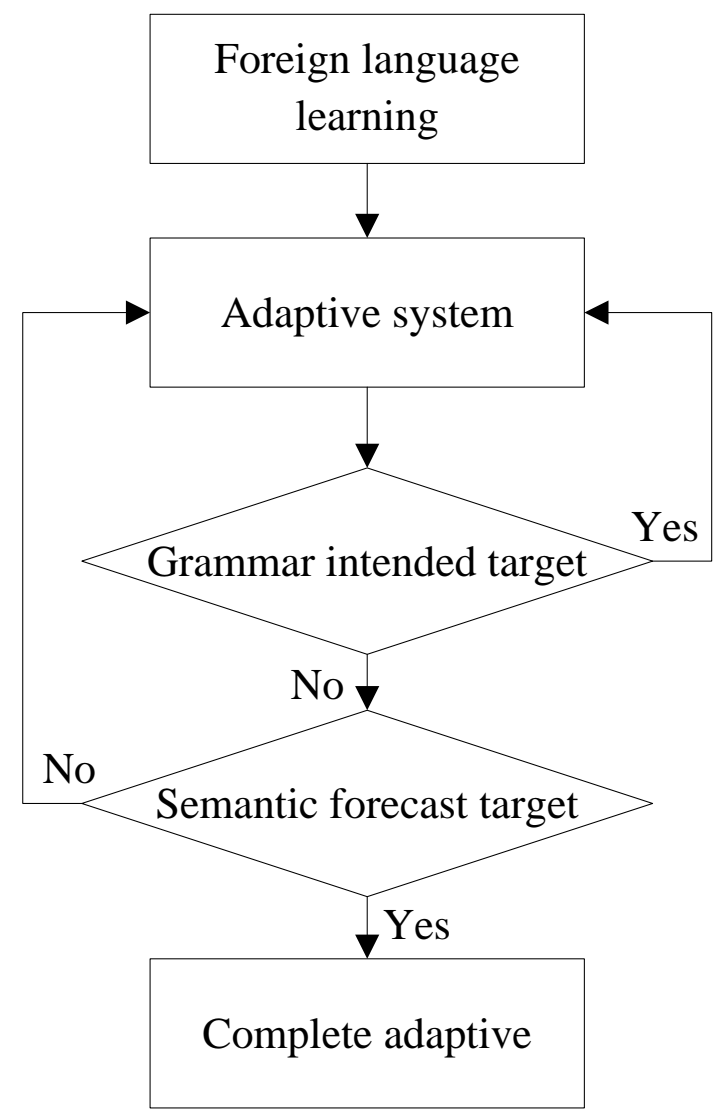

Fig. 1 The adaptive process of foreign language learning

Figure 1 shows the adaptive process of foreign language learning, in which the subject of language is human and language unit, and the main body of language is continuous self-adjustment in social exchange and interaction process, so as to realize the function of adaptation and mutual restraint, it can be reflected by the use of functional components. Each functional component is different language system, which reflects the complexity of the foreign language adaptive system.

\section{The Embodiment of Complex Adaptive Systems in Foreign Language Grammar and Semantics}

The embodiment of complex adaptive systems in empirical grammar. In the foreign language experience grammar system, the complex adaptive system will be the symbol, internal organization and module as well as the component as the main cognitive mechanism, in which the sign is the interaction between the individual and the environment, and it can be mutual recognition and selection signs through the search and receive information [12]. Internal organization and module is that the main body constantly adjusts the internal model in the process of adapting to the environment, so as to change its own organization and mechanism, to make prediction and response on the empirical grammar, and to change and adjust its structure. Component is a complex system based on the simple component, it can through changing the way of organization to carry on the transition from a simple and low level component to a complex advanced component, and it is a tool for people to understand the rules of language, which is reflected in the use of empirical grammar.

Halliday thinks that the grammar cognition of foreign language is mainly reflected by the three different phenomena of graphics, composition and sequence, its identification and construction experience are based on the category, the whole and the local as well as the ecological and functional selection, so as to express the meaning of experiential grammar. The symbolic mechanism of human foreign language cognition is embodied in the categorization cognitive mechanism of the empirical grammar, that is, the individual phenomenon is named, and the internal organization model is reflected in the graphics recognition mechanism. Through the adaptation of foreign language grammar environment and constructing the model to represent the world's experience, the subject is 
constantly changing according to the grammar environment, and makes response and forecasts for the existing empirical model, so as to adjust itself to better adapt to the experience grammar environment, impacting the whole language system changes. The individual will continue to adapt to the of the external dynamic evolution world, its component is a low level of composition, and it can form a high-level complex structure through the organization, such as language sequences are composed of graphic symbols, and graphic symbols are composed by different functional components, these ingredients are the phrases and phrases of empirical language. Therefore, component is a cognitive tool for human identification construction experience in the functional foreign language grammar.

The embodiment of complex adaptive systems in foreign language semantics. The complex adaptive system of linguistics is generated by the biological sciences, it regards language as an organism to expound the language cognition and system evolution, and it also the essence of the brain nervous system [13]. The research on the functional foreign language system is great significance in the information age, and it is to further explore the essence of the ecological evolution. As the network environment is different, the semantic experiences of individual displays are also different, leading to the language's structure, intensity, memory, and value variation, and eventually leading to the change of consciousness flow between people and people. Because the variation constitutes a different variant, the advanced consciousness can construct the adaptive model of the semantic environment through development and selection, the use of experience and judgment as well as the choice of conditions.

\section{Research on the Functional Language Learning Based on Complex Adaptive System}

In order to study the effects of complex adaptive systems on foreign language learning, a variety of stimulating channels are used to stimulate the foreign language learning, including auditory, visual and audio-visual. The preservation effect of foreign language learning memory as shown in Figure 2 is obtained by the stimulus.

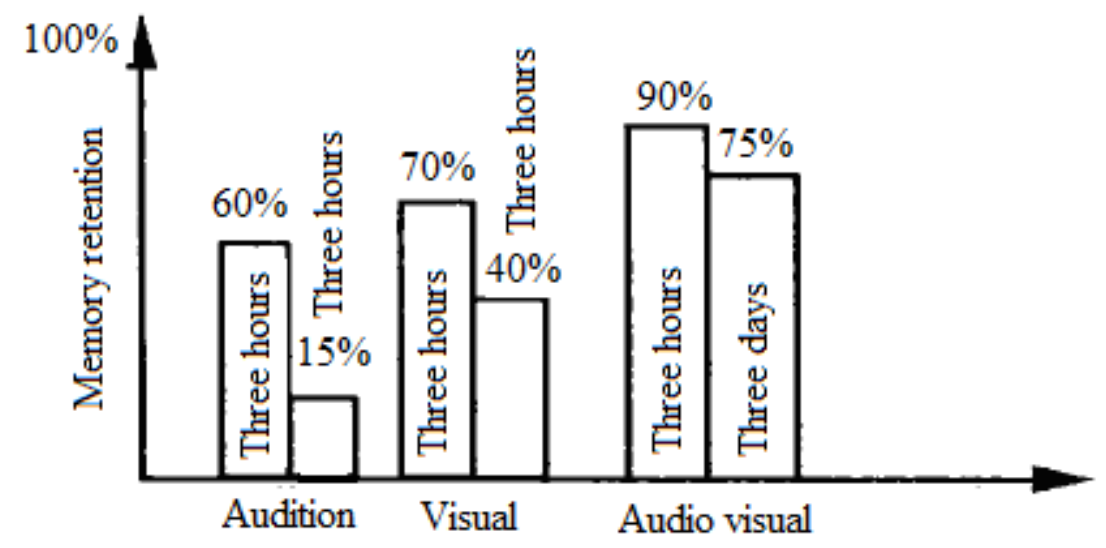

Fig. 2 The preservation effect of foreign language learning memory

Figure 2 shows the effect of foreign language learning memory, it can be seen that the multi-channel stimulation can significantly improve the learning efficiency. The research shows that using auditory alone can obtain knowledge $60 \%$ in three hours, and drop to $15 \%$ after three days; using vision alone can obtain knowledge $70 \%$ in three hours, and drop to $40 \%$ after three days; using audio-visual alone can obtain knowledge $90 \%$ in three hours, and drop to $75 \%$ after three days. Thus, the amount of language information can be obtained by the audio-visual perception, so as to obtain the best way of information acquisition. 


\section{Language adaptive level}
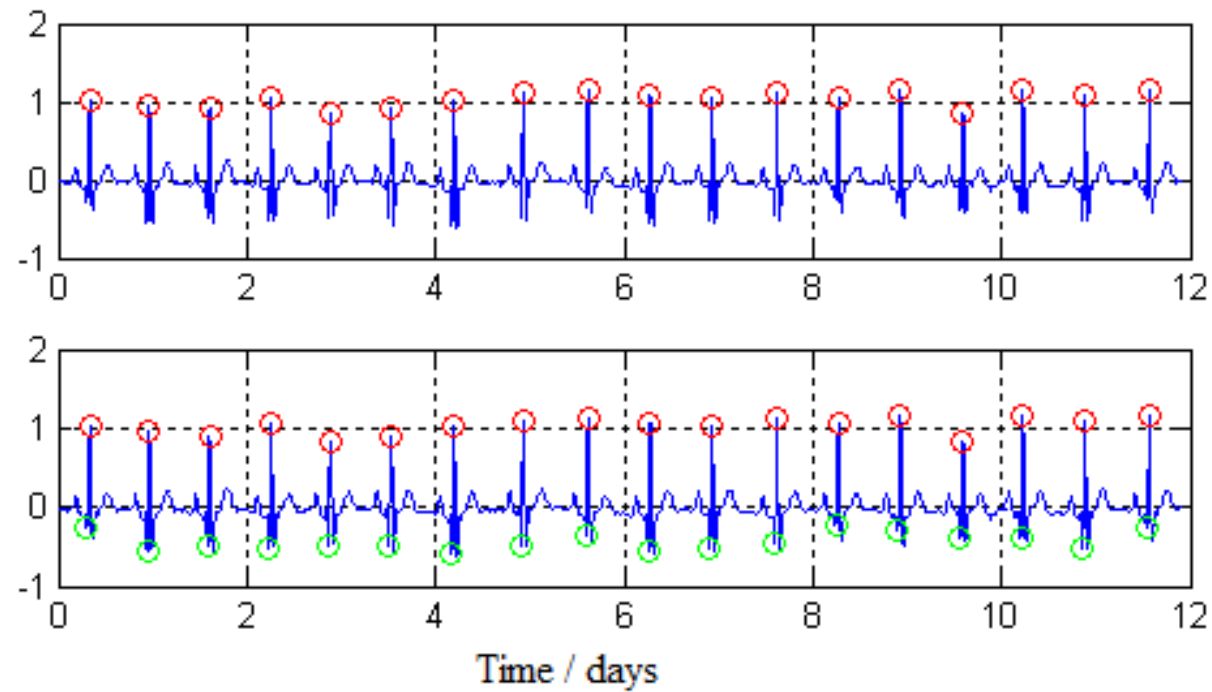

Fig.3 The adaptive horizontal curve of foreign language

Figure 3 shows the test curve of the language adaptive level, it can be seen that in the functional foreign language learning process, the adaptive level of foreign language is different in different time periods, which is due to the use of different stimulation channels for learners to stimulate, thus resulting in different effects. Therefore, in the research and teaching of foreign language, people should consider the influence of various stimulation channels on learners, as so to improve the language learners’ learning level and adaptive ability.

\section{Summary}

In order to study the influence of complex adaptive systems on foreign language linguistics, this paper deeply discusses the complex adaptive system theory in the embodiment of functional foreign linguistics, and analyzes the effects of the system on foreign language grammar and semantics. A variety of stimulating channels are used to stimulate the foreign language learning, including auditory, visual and visual-auditory, and the foreign language learning and memory preservation effect diagram is obtained. From the test results, we can get more language information through the visual-auditory, so as to obtain the best information acquisition way. By the test curve of the language adaptive level, it can be seen that using different stimulation channels have different adaptive levels of foreign language. Therefore, in the process of foreign language learning and teaching, we should consider the influence of many kinds of stimulating channels on learners, and this study will provide a more extensive research space for foreign language linguistics.

\section{References}

[1] G.W. Huang. The integration of functional linguistic research. Chinese foreign language, 2009(1): 17-23.

[2] S.Q. Yan. The origin of meaning evolution theory. Foreign language teaching and research, 2013(1): 45-53.

[3] Y.S. Zhu. The individual study of systematic functional linguistics and the philosophy guiding ideology. Modern foreign language, 2013(4): 331-337.

[4] Z.L. Hu. The diversification of context research. Foreign language teaching and research, 2014(3): 161-166.

[5] F.Y. Li. The application and thinking of multimedia in English teaching. China information technology education, 2014(8): 43-44. 
[6] L.J. Song. Analysis of middle school students' psychological characteristics in English teaching. Educational exploration, 2014(3): 55-56.

[7] J. Song. English teaching should pay attention to the students' emotion. Chapter, 2013(5): 92-93.

[8] Y.M. Zhu. A new thinking on improving students' learning interest and motivation in English teaching. China's off campus education, 2014(4): 105-106.

[9] Y. Yang. The advantages and disadvantages of multimedia teaching in English teaching and learning. Journal of Jilin radio and television university, 2013(6): 146-147.

[10] Y.H. Zhang. A study on the dynamic evaluation model of web writing teaching in college English. Foreign language circles, 2014(4): 73-81.

[11] X.Q. Wang. Higher mathematics stratified teaching mode theory and its practice. Jilin education, 2014(11): 20- 21.

[12] D.H. Mao. Research on the implementation of higher vocational mathematic hierarchical teaching. Journal of Chongqing vocational and technical college, 2014,16(4): 43- 44.

[13] B.P. Chen, A.L. Hu. Analysis of the stratified teaching mode in higher education popularization stage. Journal of Liaoning radio and TV university, 2014(2): 51- 52. 